INPLASY

PROTOCOL

To cite: Yang et al. Traditional Chinese mind-body exercises for insomnia: a protocol for systematic review and metaanalysis. Inplasy protocol 2020110078. doi:

10.37766/inplasy2020.11.0078

Received: 17 November 2020

Published: 18 November 2020

Corresponding author:

Deyu Cong

congdeyu666@sina.com

Author Affiliation:

Changchun University of

Chinese Medicine, Changchun

130117, China

Support: No: 2018YFC1706002.

Review Stage at time of this submission: Preliminary

searches.

Conflicts of interest:

None.

\section{Traditional Chinese mind-body exercises for insomnia: a protocol for systematic review and meta-analysis}

Yang, K¹; Hu, G2; Wang, Y3; Zhang, H4; Lou, H5; Cong, D6.

Review question / Objective: Insomnia is a global public problem, which has a signifificant negative impact on both physical and mental health, while increasing the economic burden placed on both sufferers and society. Due to the side effects of pharmacological therapy, people are seeking to choose complementary and alternative therapies for insomnia disorder. Traditional Chinese mind-body exercises is a representative therapy for the treatment of insomnia, and its effectiveness and safety has been validated clinically.

Condition being studied: There is a lack of systematic evaluation and analysis of traditional Chinese mind-body exercises.Therefore, the effectiveness and safety of traditional Chinese mind-body exercises in the treatment of insomnia are studied and systematically evaluated in this study.

INPLASY registration number: This protocol was registered with the International Platform of Registered Systematic Review and Meta-Analysis Protocols (INPLASY) on 18 November 2020 and was last updated on 18 November 2020 (registration number INPLASY2020110078).

\section{INTRODUCTION}

Review question / Objective: Insomnia is a global public problem, which has a significant negative impact on both physical and mental health, while increasing the economic burden placed on both sufferers and society. Due to the side effects of pharmacological therapy, people are seeking to choose complementary and alternative therapies for insomnia disorder. Traditional Chinese mind-body exercises is a representative therapy for the treatment 
of insomnia, and its effectiveness and safety has been validated clinically.

Condition being studied: There is a lack of systematic evaluation and analysis of traditional Chinese mind-body exercises. Therefore, the effectiveness and safety of traditional Chinese mind-body exercises in the treatment of insomnia are studied and systematically evaluated in this study.

\section{METHODS}

Participant or population: Studies will be eligible if they include participants with a diagnosis of insomnia. There were no limitations on age, gender, or nationality of patients with insomnia.

Intervention: In this review, traditional Chinese mind-body exercises include Tai Chi, Qigong, Baduanjin, Wuqinxi, and Yijinjing. The included studies use one of them to treat insomnia. The control interventions include medicine, observation, manual therapy, acupuncture, traction, education, and any treatments without traditional Chinese mind-body exercises. The study assessing the effects of traditional Chinese mind-body exercises plus an intervention compared with the same intervention (such as Tai Chi plus traction versus traction) will also be included.

Comparator: Study designs to be included: his review will only include RCTs of traditional Chinese mind-body exercises for insomnia. Case reports, observational studies, and cross-sectional design studies will be excluded. The study protocol and conference abstract of RCTs will also be excluded, if the corresponding author could not provide detailed information.

Study designs to be included: This review will only include RCTs of traditional Chinese mind-body exercises for insomnia. Case reports, observational studies, and cross-sectional design studies will be excluded. The study protocol and conference abstract of RCTs will also be excluded, if the corresponding author could not provide detailed information.

Eligibility criteria: Studies will be eligible if they include participants with a diagnosis of insomnia. There were no limitations on age, gender, or nationality of patients with insomnia.

Information sources: The electronic databases (PubMed, Embase, Web of Science, Cochrane Library, China Knowledge Resource Integrated Database, and Wanfang Data) will be searched from their inception to December 2020. Two sets of search terms will be used, as follows: (1) "Chronic Insomnia"or"Primary Insomnia"or"Secondary Insomnia" or"Comorbid Insomnia"or"Disorder of Initiating and Maintaining Sleep"or "Behavioral Insomnia of Childhood "or "Sleep-onset Association Disorder" or "Limit-setting Sleep Disorder";[22]and (2) "Tai Chi" or "Tai Chi Chuan" or "Qigong" or "Baduanjin" or "Wuqinxi" or "Yijinjing".

Main outcome(s): The primary outcome includes Pittsburgh sleep quality index and clinical effective rate. Pittsburgh sleep quality index is comprised of 19 self-rated items in 7 factors and the total scores range from 0 to 21.Clinical effective rate is calculated based on the criteria for the therapeutic effects of western medicine and traditional Chinese medicine, according to Guiding Principles for Clinical Research of New Chinese Medicine. Clinical recovery: sleep time has returned to normal or the sleep time at night has been more than 6 hours, deep sleep and energetic after waking up. Markedly effective: sleep has improved signifificantly, sleep time has increased by more than 3 hours, and sleep depth has increased. Effective: the symptoms have relieved, but the sleep time increases less than 3 hours longer than before. Invalid: no improvement or aggravation of insomnia. The total effective rate $=$ (Clinical recovery +Markedly effective + Effective)/Total number of cases $100 \%$. 
Quality assessment / Risk of bias analysis: The quality assessment of the included studies will be independently conducted by two reviewers using the Physiotherapy Evidence Database (PEDro) scale. The PEDro scale is a tool developed to measure the methodological quality of RCTs of physiotherapy interventions. The scale involves an 11-domain assessment: 1) study eligibility criteria specified, 2) random allocation of subjects, 3) concealed allocation, 4) measure of similarity between groups at baseline, 5) subject blinding, 6) therapist blinding, 7) assessor blinding, 8) less than $15 \%$ dropouts, 9) intention- totreat analysis, 10) between-group statistical comparisons, and 11) point measures and variability data. The PEDro score will be calculated by criteria 2) to 11) according to meeting the criteria or not. From these scores, the studies are considered as excellent (9-10 points), good (6-8 points), fair (4-5 points), and poor (less than 4 points) quality.

Strategy of data synthesis: The metaanalysis will be conducted using Review Manager Version 5.3 software. For continuous data, the change between baseline and the end of interventions will be used in the meta-analysis. The mean difference (MD) and $95 \%$ confidence intervals $(\mathrm{Cl})$ will be calculated. In the case of different outcome measure scales, the standardized mean difference (SMD) and 95\% Cl will be calculated. For the expected heterogeneity, the continuous data will be pooled using a more conservative randomeffects model.

Subgroup analysis: If considerable heterogeneity is found, subgroup analysis would be envisaged to perform to investigate probable sources of heterogeneity in accordance with the duration of treatment, age, history of insomnia, and research quality.

Sensibility analysis: In order to ascertain the sensitiveness of results to modifications in initial assumptions, the authors will conduct sensitivity analysis by only including studies with low risk of bias.
Country(ies) involved: China.

Keywords: Traditional Chinese mind-body exercises, Insomnia, Meta-analysis, Systematic review

Contributions of each author:

Author 1 - Kang Yang.

Author 2 - Guanyu Hu.

Author 3 - Yufeng Wang.

Author 4 - Hongshi Zhang.

Author 5 - Huijuan Lou.

Author 6 - Deyu Cong. 